\title{
Outcomes of sphincterotomy for small pupil phacoemulsification
}

\author{
Joanne W Y Goh ${ }^{1} \cdot$ Rhys Harrison ${ }^{1} \cdot$ Shokufeh Tavassoli $^{1} \cdot$ Derek M Tole $^{1}$ \\ Received: 3 August 2017 / Revised: 13 January 2018 / Accepted: 8 February 2018 / Published online: 5 April 2018 \\ (c) The Royal College of Ophthalmologists 2018
}

\begin{abstract}
Purpose Sphincterotomy, an alternative to iris hooks or pupil stretching, is a technique that can aid in small pupil phacoemulsification. The incidence of post-operative complications of this procedure, however, has not been studied. Our study evaluates the post-operative outcomes of phacoemulsification surgery with adjunctive pupillary sphincterotomy.

Methods We conducted a retrospective review of case notes and Medisoft ${ }^{\circledR}$ electronic record of patients that had undergone simultaneous sphincterotomy, phacoemulsification and intraocular lens (IOL) implantation by a single surgeon between March 2012 and February 2017. Our main outcome measures were post-operative ocular hypertension (IOP $>21 \mathrm{mmHg}$ ), uveitis and cystoid macular oedema (CMO).

Results A total of 114 eyes of 114 patients were included in this study. The mean age was 81.2 years (range: 26-100). All patients had uncomplicated surgery. Transient ( $<1$ month) ocular hypertension developed in five (4\%) eyes. Sustained ocular hypertension (>1 month) occurred in one (1\%) eye, which had pre-existing glaucoma. All four (4\%) eyes that developed a persistent uveitis (>1 month) resolved with topical therapy except for one eye with a history of uveitis. The $6(5 \%)$ eyes that developed CMO had a history significant for uveitis $(n=4)$, diabetic macular oedema $(n=1)$ and epiretinal membrane $(n=$ 1). All CMO maculae resolved to their baseline.

Conclusion The incidence of post-operative complications following uncomplicated phacoemulsification and IOL implantation with pupillary sphincterotomy is low. The most important predisposing factors for development of a complication are ocular co-morbidities such as glaucoma, uveitis and the presence of a macular pathology.
\end{abstract}

\section{Introduction}

A pupil that does not dilate adequately for cataract surgery requires enlargement with one of several techniques to allow surgery to be carried out safely. Early reports have described surgical techniques such as superior sector iridectomy [1], superior and/or inferior radial iridotomy [2], multiple sphincterotomies [3], inferior sphincterotomy with a preplaced suture [4], and iris retraction with a spatula or suture to surgically stretch the pupil [5]. These techniques, however, have become almost redundant with the introduction of new devices such as iris hooks, rings and pupil expanders because of their safety and ease of use. The main drawback of these devices, however, is their cost.

The technique of creating multiple sphincterotomies and stretching the sphincterotomies to enlarge an inadequately

Joanne W Y Goh

joanneyau.goh@gmail.com

1 Bristol Eye Hospital, Bristol, UK dilated pupil for phacoemulsification surgery was first described by Fine [3]. This technique is a useful alternative when iris hooks and other pupil expanders are not available. The pupil has been reported to recover excellent physiologic function and cosmesis postoperatively;[3] however, there are no studies examining the safety of this procedure.

In this study, we describe our modified sphincterotomy technique and evaluate the incidence of postoperative complications of this procedure when used in small pupil phacoemulsification.

\section{Methods}

The Bristol Eye Hospital institutional audit committee reviewed and approved this study. A retrospective study of all patients who had sphincterotomy, phacoemulsification and intraocular lens (IOL) implantation performed by a single surgeon (DMT) between March 2012 and Feb 2017 was conducted. Data on each patient's ocular history, past medical history and postoperative outcome measures i.e. intraocular pressure (IOP), anterior chamber activity and 
Table 1 Baseline characteristics of patients included in study

Patient characteristics

\begin{tabular}{ll}
\hline Average age (years) & 81.2 (range: 26-100) \\
Diabetes, $n(\%)$ & $21(18 \%)$ \\
Alpha-blocker medication, $n(\%)$ & $35(31 \%)$ \\
Macula pathology, $n(\%)$ & $28(25 \%)$ \\
Epiretinal membrane & $5(4 \%)$ \\
Dry ARMD/GA & $18(16 \%)$ \\
Neovascular AMD & $3(3 \%)$ \\
Diabetic macula oedema & $1(<1 \%)$ \\
Previous history of uveitis, $n(\%)$ & $9(8 \%)$ \\
Glaucoma/OHT, $n(\%)$ & $13(11 \%)$ \\
\hline
\end{tabular}

presence of cystoid macular oedema (CMO) at the first and subsequent follow-up visits was collected from the case notes and Medisoft (Leeds, UK) electronic patient record.

\section{Surgical technique}

Preoperatively, pupils are dilated pharmacologically with cyclopentolate $1 \%$, phenylephrine $2.5 \%$ and tropicamide $1 \%$ at $15 \mathrm{~min}$ intervals $1 \mathrm{~h}$ before surgery. All patients received a subtenons local anaesthetic injection consisting of a 50:50 mixture of $2 \mathrm{ml} 2 \%$ lignocaine and $2 \mathrm{ml} 0.5 \%$ bupivacaine with $1 / 200,000$ adrenaline. Intra-operatively, 2.5\% phenylephrine diluted in a 50:50 mixture of balanced salt solution was injected intracamerally to attempt further pupil dilation. If inadequate pupil dilation is achieved, multiple sphincterotomies are performed after the anterior chamber has been filled with viscoelastic.

Approximately 12-16 radial sphincterotomies measuring approximately $0.50 \mathrm{~mm}$ limited to the iris sphincter are cut at equal intervals around the iris border using a 23G Prasad scissors (Optico, Herts, UK). The scissors are narrow enough to fit through a $1.2 \mathrm{~mm}$ paracentesis. The sphincterotomies are performed on the temporal half of the pupillary border via the nasal paracentesis and completed on the nasal half via the temporal paracentesis. This results in a dramatic increase in pupil size. After performing the sphincterotomies, the anterior chamber is deepened with Healon GV (Abbott Vision, Illinois, USA), resulting in further pupil dilation, typically $6.0 \mathrm{~mm}$ or more in diameter, through which cataract extraction can be easily performed. After phacoemulsification and lens implantation, residual viscoelastic is removed, intracameral acetylcholine chloride (Miochol, Bausch and Lomb, Surrey, UK) and cefuroxime (Aprokam, Thea, Clermont-Ferrand Cedex, France) are instilled, and a subconjunctival injection of betamethasone is given. At the conclusion of the surgery, the pupil is usually 3.0 to $4.0 \mathrm{~mm}$ in diameter.
For the first 4 weeks, all patients used an antibiotic drop four times a day for 1 week and a steroid drop (dexamethasone $0.1 \%$ ) every $2 \mathrm{~h}$ for the first 2 postoperative weeks which is then tapered down to four times a day at the 2 -week postoperative visit if the eye is quiet.

\section{Results}

A total of 125 eyes from 120 patients underwent simultaneous sphincterotomy, phacoemulsification and IOL implantation between March 2012 and February 2017. For bilateral cases, only one eye was selected at random. Six cases were excluded due to incomplete follow up data. A total of 114 eyes from 114 patients were included in this study. Table 1 shows the characteristics of the eyes in this cohort. The average age of the patients was 81.2 years (range: 26-100 years). Almost one-fifth of the patients whose eyes were operated on had diabetes and approximately a third were on an $\alpha$-blocker. The prevalence of any preoperative macular pathology was $25 \%$; these pathologies included epiretinal membranes (ERM), age-related macular degeneration (AMD), and diabetic macular oedema (DMO). A history of uveitis and glaucoma was present in $8 \%$ and $11 \%$ of the eyes, respectively. The average time for the first follow-up visit was 23 days and the average complete follow up duration was 92 days.

All patients underwent uncomplicated cataract surgery. Despite the iris incisions, no cases of iris bleeding occurred intraoperatively. The frequency of postoperative complications is presented in Table 2. Transient ocular hypertension, defined as an IOP greater than $21 \mathrm{mmHg}$ lasting for less than a month, occurred in $5(4 \%)$ eyes at a mean follow-up of 14 days, of which none had underlying glaucoma. Sustained ocular hypertension, defined as an IOP greater than $21 \mathrm{mmHg}$ persisting beyond 1 month, was seen in 1 patient (1\%), who had pre-existing glaucoma. This patient settled to baseline without any additional IOP lowering therapy.

Table 2 Frequency of postoperative complications following uncomplicated sphincterotomy, cataract extraction and intra-ocular lens implant

\begin{tabular}{ll}
\hline Complications & Numbers \\
\hline Ocular hypertension & $6(5 \%)$ \\
Transient $(<1$ month) & $5(4 \%)$ \\
Sustained (>1 month) & $1(<1 \%)$ \\
Persistent uveitis ( $>1$ month) & $4(4 \%)$ \\
Cystoid Macula Oedema & $6(5 \%)$ \\
Corneal Oedema & $10(9 \%)$ \\
Transient $(<1$ month) & $9(8 \%)$ \\
Sustained (>1 month) & $1(<1 \%)$ \\
\hline
\end{tabular}


Persistent uveitis, defined as the presence of anterior chamber cells one month after surgery, was documented in $4(4 \%)$ eyes. This resolved with topical steroid therapy in all but one eye that had a history of HLA-B27 related chronic uveitis controlled on a long-term topical steroid, and did return to baseline after an 8-week course of steroids.

$\mathrm{CMO}$ occurred in six (5\%) eyes, of which four eyes had a history of uveitis, one had previous diabetic macular oedema and another had an ERM. All cases of CMO resolved with topical therapy alone. One eye developed two complications; CMO and persistent uveitis. This patient had a history of diabetic macula oedema, previous macula laser and ERM and did eventually return to his preoperative baseline. The average time for the diagnosis of CMO 21 days.

Nine eyes developed transient corneal oedema in the first month and one eye, which had a brunescent cataract developed persistent corneal oedema ( $>1$ month) requiring subsequent endothelial keratoplasty. Of the eyes which developed corneal oedema three had co-existent Fuch's endothelial corneal dystrophy, four had dense or brunescent cataracts and two had narrow angles.

All post sphincterotomy eyes recovered excellent cosmesis, dilated adequately for fundoscopy and were able to respond physiologically to light and dark (Fig. 1).

\section{Discussion}

Sphincterotomies can be safely employed to perform successful cataract surgery in eyes with small pupils. This technique consistently produces a pupil with a diameter adequate for phacoemulsification in a time-efficient manner and results in a cosmetically acceptable and physiologically functional pupil postoperatively. Our study showed that patients without preoperative glaucoma had no significant risk of postoperative ocular hypertension. A mild persistent uveitis may occur in some eyes, requiring a longer duration

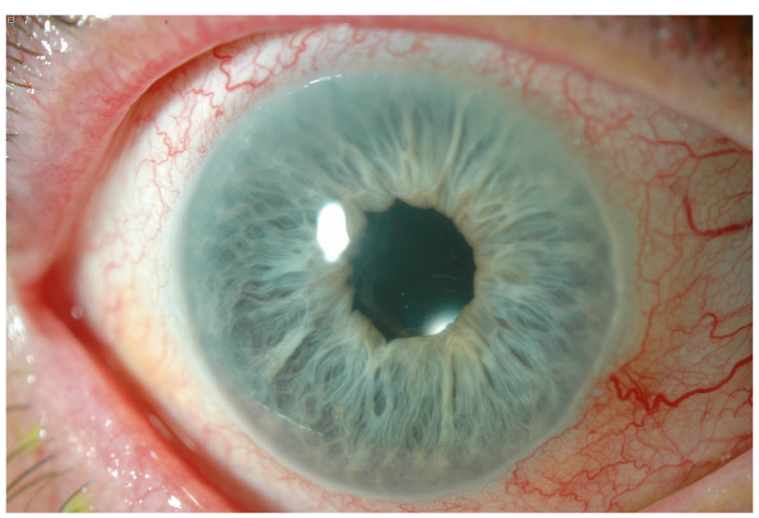

Fig. 1 Postoperative appearance of an eye that underwent sphincterotomy, phacoemulsification and intra-ocular lens implantation of topical steroid therapy. Iris manipulation may trigger exudative transudation into the anterior chamber in predisposed eyes, thereby causing an exacerbation or reactivation of uveitis in eyes with a prior history, as seen in our case with HLA-B27. In our study, CMO only occurred in eyes with previous uveitis, DMO, and ERM, all of which are independent risk factors for postoperative CMO. Further studies are needed to clarify this association with sphincterotomies.

The creation of multiple iris incisions can be expected to increase the incidence and severity of postoperative uveitis leading to ocular hypertension and $\mathrm{CMO}$; however, the risk of these occurring was not significantly increased. The administration of subconjunctival steroids and the use of frequent topical steroid drops postoperatively are plausible mitigating explanations for the incidence of $\mathrm{CMO}$ and uveitis; however, could also account for the occurrence of mild, self-limiting ocular hypertension seen in $4 \%$ of our cases.

Sphincterotomies can be used in most patients in whom the pupil is inadequate for safe phacoemulsification and IOL implantation surgery. Sphincterotomies are safe, quick and easy to perform, requires little instrumentation, and yields a consistently dilated pupil so that phacoemulsification can be safely performed. Unlike pupil stretching, intraoperative miosis does not occur with sphincterotomies. Sphincterotomies also do not require surgical repair unlike other surgical techniques such as superior sector iridectomy and superior and/or inferior radial iridotomy, which require a pupilloplasty at the end of surgery to avoid disabling postoperative glare and diplopia.

The strengths of this study include having a large cohort of patients whose surgeries were performed by a single surgeon, thereby eliminating operative variabilities affecting the outcome measures. However, a potential limitation of this study is its retrospective design; hence, we are only able to draw associations but are unable to determine causal and temporal relationships. Additionally, selection bias cannot be avoided in this cohort of patients, and because we are limited to analysing pre-existing data that has been collected, the results may be subject to confounding wherein not all potential risk factors could be assessed as they had not been measured.

In summary, our study shows that sphincterotomies are a safe aid to small pupil phacoemulsification surgery. Sphincterotomies are quick and simple to perform and maintains a constant mydriasis, facilitating uniform visualisation throughout surgery. The risk of post-operative complications is low, and may be influenced by the presence of a predisposing factor such as glaucoma, uveitis or a macular pathology. 


\section{Summary}

\section{What was known before}

- Sphincterotomies can be used as an aid in small pupil phacoemulsification as it produces a pupil of adequate diameter for safe phacoemulsification.

- The safety and incidence of post-operative complications, however, has not been studied.

\section{What this study adds}

- We evaluated the post-operative outcomes of phacoemulsification surgery with adjunctive pupillary sphincterotomy.

- We found that the incidence of post-operative complications is low; however, this risk may be influenced by the presence of a predisposing factor such as uveitis, glaucoma or a macular pathology.

\section{Compliance with ethical standards}

Conflict of interest The authors declare that they have no conflict of interest.

\section{References}

1. Cole MD, Brown R, Ridgway AE. Role of sphincterotomy in extracapsular cataract surgery. Br J Ophthalmol. 1986;70:692-5.

2. Mackool RJ. Small pupil enlargement during cataract extraction. A new method. J Cataract Refract Surg. 1992;18:523-6.

3. Fine IH. Pupilloplasty for small pupil phacoemulsification. J Cataract Refract Surg. 1994;20:192-6.

4. Masket S. Preplaced inferior iris suture method for small pupil phacoemulsification. J Cataract Refract Surg. 1992;18:518-22.

5. Freeman WR, Feldman ST, Munguia D, Mendez T, Wiley CA. The prethreaded pupillary dilating (torpedo) suture for phakic and aphakic eyes. Arch Ophthalmol. 1992;110:564-7. 